\title{
Las consecuencias de la aplicación del Real Decreto-Ley de Regulación Sanitaria RD 16/2012 sobre la salud de las mujeres inmigradas
}

\author{
Anna MORERO BELTRÁN \\ anna.morero@ub.edu \\ Ana BALLESTEROS PENA \\ a.ballesterospena@gmail.com \\ GRC Copolis, Benestar, Comunitat i Control social \\ Universitat de Barcelona
}

Recibido: 07.09.2014

Aceptado: 21.01.2015

\begin{abstract}
RESUMEN
El Real Decreto-Ley 16/2012, de 20 de abril, de "Medidas urgentes para garantizar la sostenibilidad del Sistema Nacional de Salud y mejorar la calidad y seguridad de sus prestaciones", supuso la eliminación del derecho al acceso a la salud para las personas no inscritas en el Régimen de la Seguridad Social, poniendo así punto y final a la atención sanitaria gratuita y universal para toda la ciudadanía del Estado español. Como consecuencia de la aplicación de este Real Decreto-ley se ha generado un acceso desigual a un derecho humano básico y legalmente reconocido. Este artículo pretende ofrecer un diagnóstico del impacto de la eliminación de dicha atención en las personas inmigradas, concretamente en lo que se refiere a las mujeres, y de las consecuencias derivadas del Real Decreto-ley en materia de violencia contra las mujeres y de derechos sexuales y reproductivos. Además, este artículo tiene también como objetivo recoger el trabajo realizado por las organizaciones y plataformas surgidas para la reivindicación y la defensa del derecho a la salud. En ambos casos, se abordará de manera particular el caso de Cataluña.
\end{abstract}

Palabras clave: Salud sexual, Salud reproductiva, Mujeres, Inmigración, Real Decreto-Ley 16/2012

\section{The consequences of the implementation of the Royal Decree-Law of sanitary regulation $R D$ 16/2012 on migrant women's health.}

\begin{abstract}
The Royal Decree-Law 16/2012 for sanitary regulation, led to the elimination of certain benefits for people not registered in the Spanish National Security Social System. Thus, this means the end of the universal and free health care for all citizens who live in Spain. This recent regulation creates an unequal access to a recognized basic human right. This paper aims to provide an assessment of the impact of the elimination of such health care rights for immigrant people, specifically by exploring their implications for immigrant women, and the consequences of the implementation of the Royal Decree-Law on violence against women and on sexual and sexual and reproductive rights. In addition,
\end{abstract}


this paper also seeks to collect the efforts of organizations who work in defense and for the vindication of the universality of the health in Spain. This paper will address, particularly, the case of Catalonia.

Key words: Sexual health, Reproductive health, Women, Immigration, Royal Decree-Law 16/2012

\section{INTRODUCCIÓN ${ }^{1}$}

El pasado mes de noviembre de 2013, la Relatora Especial de Naciones Unidas sobre la extrema pobreza y los derechos humanos, Magdalena Sepúlveda, en una carta enviada al gobierno español junto con otros expertos en derechos humanos de la ONU, advertía de que "los cambios adoptados por el Gobierno de España en 2012, que reducen el acceso al sistema de salud pública, no cumplen con las obligaciones de derechos humanos del país". Los cambios a los que se refiere la Relatora son los contemplados en el Real Decreto-ley 16/2012 $2^{2}$, denominadode medidas urgentes para garantizar la sostenibilidad del Sistema Nacional de Salud y mejorar la calidad y seguridad de sus prestaciones, que entre otras medidas limitala asistencia sanitaria para las personas inmigradas mayores de edad en situación administrativa irregular al servicio de urgencias y a la asistencia durante el embarazo, parto y postparto.

Las advertencias de la Relatora fueron reiteradas el pasado 29 de abril de 2014 en un comunicado de prensa ${ }^{3}$ en el que insistía en la necesidad de que las autoridades españolas garantizasen el acceso universal a la salud. Además de reprobar este RDL 16/2012 porque atenta contra los derechos humanos y supone un elevado coste humano, la relatora añadió que lo considera una medida poco efectiva para reducir los costes a largo plazo del sistema sanitario. En este sentido, indicó que la falta de acceso a la atención primaria supone una sobrecarga de los servicios

\footnotetext{
${ }^{1}$ A lo largo del artículo las denominaciones inmigrantes, migrantes, población extranjera inmigrante, mujeres migradas se utilizarán como sinónimos, a pesar de que las autoras son conscientes de los diferentes matices que se pueden derivar de la utilización de uno u otro término.

${ }^{2}$ Con el fin de no resultar reiterativas, a lo largo del texto nos referiremos al Real DecretoLey 16/2012 de medidas urgentes para garantizar la sostenibilidad del Sistema Nacional de Salud y mejorar la calidad y seguridad de sus prestaciones con las siglas RDL 16/2012. Idéntica estrategia se utilizará con otra normativa de la misma naturaleza, una vez se haya incluido su denominación completa la primera vez.

${ }^{3}$ Disponible en :

http://www.ohchr.org/SP/NewsEvents/Pages/DisplayNews.aspx?NewsID=14545\&LangID= $\mathrm{S}$
} 
de emergencia, más costosos, y, por otro lado, advirtió de los posibles riesgos para la salud pública ${ }^{4}$.

En idéntico sentido, el Comité Europeo de Derechos Sociales, órgano del Consejo de Europa encargado de velar por el cumplimiento de la Carta Social Europea, sentenciaba, a inicios también del año 2014, que el Estado español no respeta la Carta en lo que se refiere al derecho a la salud, desde que se aprobara el RDL 16/2012.

Por tanto, como mostraremos a lo largo del artículo, esta normativa supone una vulneración efectiva del derecho a la protección de la salud; un derecho humano reconocido desde la Constitución de la Organización Mundial de la Salud (OMS) y por la Organización de las Naciones Unidas (ONU) en la Declaración Universal de los Derechos Humanos. Numerosos tratados y normativas internacionales, como por ejemplo la Carta Social Europea o la Declaración de Alma Ata, reconocen la protección de la salud como un derecho fundamental y que corresponde a los Estados proteger. Además el RDL 16/2012 infringe el Pacto Internacional sobre derechos económicos, sociales y culturales (PIDESC), uno de los tratados más importantes en esta materia, ratificado también por España.

Cuando la implantación de la reforma ya ha cumplido dos años, este artículo pretende realizar un análisis sobre sus implicaciones en las mujeres inmigradas de forma particular, abordando sus consecuencias en la prevención, detección y atención de casos de violencia machista, así como sus implicaciones sobre los derechos sexuales y reproductivos, concretamente en el acceso a la interrupción voluntaria del embarazo, con especial atención al caso de Cataluña. Para ello se ha realizado una revisión bibliográfica, así como un análisis del trabajo llevado a cabo por diversas organizaciones, grupos vecinales y plataformas de profesionales que, a través de diversas estrategias, tratan de paliar las graves consecuencias de esta reforma.

\section{LA APLICACIÓN DEL REAL DECRETO EN EL ESTADO ESPAÑOL, EL INICIO DEL APARTHEID SANITARIO.}

${ }^{4}$ Oficina del Alto Comisionado de las Naciones Unidas para los Derechos Humanos http://www.ohchr.org/SP/NewsEvents/Pages/DisplayNews.aspx?NewsID=14545\&LangID= $\underline{\text { S }}$ (Accedido el 15 de junio de 2014) 
El 20 de abril de 2012, el Gobierno del Estado español aprobó el RDL 16/2012 que modificaba la Ley de Extranjería ${ }^{5}$ y en el que se negaba el acceso a los servicios de atención a la salud y prevención principalmente a las personas inmigradas en situación administrativa irregular. Esta nueva normativa, que entró en vigor el pasado 1 de septiembre de 2012, solamente garantiza la atención gratuita en casos de urgencia, de atención al embarazo, parto y postparto, así como de las personas menores de 18 años. El resto de la población inmigrada en situación administrativa irregular queda excluida de la atención sanitaria, hasta entonces gratuita y accesible para todas las personas residentes en el Estado español ${ }^{6}$.

Inicialmente, el Gobierno justificó la adopción del RDL 16/2012 por la necesidad de reducir el gasto público, aunque posteriormente el Ministerio de Sanidad cambió su argumentario, debido a los contundentes argumentos mostrados por las organizaciones de derechos humanos que demostraban que esta normativa no supondría ningún ahorro sino todo lo contrario, ya que la desatención en estadios tempranos de cualquier enfermedad supondría una atención de urgencia posterior más costosa en términos económicos. El gobierno introduce entonces la idea de quela nueva normativa no había sido creada por razones económicas, sino que se trataba de la aplicación de lanormativa europea y las conclusiones del Tribunal de Cuentas, con el objetivo de asegurar "el principio de reciprocidad" cuando la población española viaja al extranjero y "frenar el turismo de salud" ${ }^{7}$.El discurso del turismo sanitario y los abusos por parte de la población inmigrante es replicado también por otros responsables políticos autonómicos del mismo color político del gobierno. En este sentido, y tal y como recoge un informe de Yo sí Sanidad Universal de 2013, se han podido observar declaraciones de personas integrantes del gobierno, como por ejemplo, las vertidas por José Ignacio Echániz, Consejero de Sanidad de Castilla-La Mancha, que afirmaba que "no hay que olvidar que la sanidad gratuita ha sido fundamental para fomentar el efecto llamada. Mucha gente ha cogido una patera porque sabía que en España tenía la asistencia sanitaria garantizada" (El Mundo, 23 de abril 2012). A partir de este tipo de discursos lo que

${ }^{5}$ Ley Orgánica 4/2000, de 11 de enero, sobre derechos y libertades de los extranjeros en España y su integración social: http://www.boe.es/boe/dias/2012/04/24/pdfs/BOE-A-20125403.pdf

${ }^{6}$ Otras casos de exclusión que afectan de manera intensa a la población inmigrada en nuestra país se encuentran descritos en detalle en el informe "Un año de exclusión sanitaria, un año de desobediencia" de Yo sí Sanidad Pública que se encuentra en línea:

yosisanidaduniversal.net/media/blogs/materiales/DossierAniversarioRDL.pdf

${ }^{7}$ Conferencia de prensa de la vicepresidenta y portavoz del Gobierno, y de los ministros de Educación y Sanidad, después de la reunión del Consejo de Ministros. Madrid, viernes, 20 de abril de 2012.

http://www.lamoncloa.gob.es/consejodeministros/ruedas/Paginas/2012/cmrp20120420.aspx 
se pretendía era, en primer lugar, fomentar el desinterés de la población general, ya que daba a entender que la reforma sanitaria sólo afecta a las personas inmigradas con menos recursos económicos y que no tendrá un impacto sobre el resto de la población. Y en segundo lugar, este tipo de declaraciones fomentan la idea de la existencia de un turismo sanitario financiado por todas las personas españolas, que supone un gasto a erradicar (Yo sí Sanidad Pública, 2013).

No obstante, la retirada de las tarjetas sanitarias, recogida en este Real Decreto y cifrada en el Plan de Reformas de 2013 en 873.000 personas extranjeras, teóricamente, no residentes en España ${ }^{8}$, no ha sido precedida de un análisis de la implicación de la medida en el presupuesto español, en términos económicos. Hasta el día de hoy, según informa Médicos del Mundo (2014), el Ministerio de Sanidad, Servicios Sociales e Igualdad no ha informado sobre cuál es la base de cálculo de las cifras de tarjetas retiradas; ni ha ofrecido un desglose de la información para conocer cuántas de esas tarjetas correspondían a la ciudadanía de Estados miembros de la Unión Europea, ni cuántas correspondían a personas procedentes de países tradicionalmente migratorios.

En opinión de las organizaciones ciudadanas y profesionales, como Médicos del Mundo (2014) se está originando lo que han denominado como un "apartheid sanitario". En la misma línea, la European Union Agency for Fundamental Rights, señala que su puesta en marcha ha dado lugar a un conjunto de situaciones de vulneración del derecho a la salud en el Estado español.

\subsection{La arbitrariedad de la aplicación del RDL 16/2012: diferentes comunidades autónomas, diferente aplicación.}

En virtud de la información analizada hasta el momento, hemos podido observar cómo la implementación de la nueva normativa en materia sanitaria ha sido diferente en cada comunidad autónoma, dado que estas tienen transferidas las competencias en materia de asistencia sanitaria. Por tanto, el panorama actual es muy diverso y, mientras que algunas comunidades autónomas como Andalucía y Asturias, con diferente color político que el gobierno central, rechazan la aplicación del RDL 16/2012 y llevan a cabo acciones contra el mismo, otras lo han

\footnotetext{
${ }^{8}$ Respecto a la retirada de tarjetas, y según información de Médicos del Mundo (2013), en la sesión de la Comisión de Sanidad de enero de 2014, se informaba de la baja de 676.000 personas extranjeras que ya no constan como residentes en nuestro país,mientras que en un escrito dirigido a varios mecanismos de derechos humanos de Naciones Unidas, en ese mismo mes, se informa de que han sido 748.835 las tarjetas que se han dado de baja.
} 
implementado en su totalidad, como por ejemplo es el caso de Madrid, con gobierno de idéntico signo que el ejecutivo estatal.

Entre la diversidad de aplicaciones se encuentran diez comunidades autónomas que han dispuesto medidas para proporcionar la tarjeta sanitaria a las personas inmigradas en situación administrativa irregular: Andalucía, Aragón, Asturias, Canarias, Cantabria, Cataluña, Extremadura, Galicia y Comunidad Valenciana. De estas, ocho lo hacenen forma de "programa especial" específico para las personas no aseguradas ni beneficiarias sin recursos: Aragón, Canarias, Cantabria, Cataluña, Extremadura, Galicia y Comunidad Valenciana (Médicos del Mundo, 2014).

En este marco de diversidad en la implementación de la normativa, se observan, y así lo denuncian los colectivos y organizaciones sociales ${ }^{9}$, algunos hechos comunes como son, por ejemplo, la facturación en urgencias y las dificultades en la atención a mujeres embarazadas y menores de edad. La heterogeneidad de las actuaciones emprendidas por las comunidades autónomas genera, por tanto, disparidades y desigualdades en el acceso a la salud dentro del territorio estatal, sin que hasta el momento exista ninguna valoración en términos de costes para la salud de la ciudadanía en general por parte del gobierno del Estado.

Tomando como ejemplo el caso de Cataluña, el gobierno de la Generalitat creó y aplicó la Instrucció 10/2012 del Catsalut ${ }^{10}$ que, supuestamente, ampliaba la cobertura del RDL 16/2012. Concretamente, el Departament de Saluta través de la instrucción ofrece cobertura a aquellas personas en situación administrativa irregular que estén empadronadas un mínimo de tres meses continuados en alguna localidad catalana. Se establecen dos modelos de acceso que son los siguientes: el correspondiente al primer nivel de asistencia sanitaria, vinculado a personas

${ }^{9}$ MÉDICOS DEL MUNDO lo indica en su informe de septiembre de 2013 El informe de la reforma sanitaria en el derecho a la salud, accesible en:http://www.medicosdelmundo.org/derechoacurar/wp-content/uploads/2012/11/Resumendel-impacto-de-la-reforma-sanitaria-en-el-derecho-a-la-salud-de-la-poblaci\%C3\%B3ninmigrante-Casos-documentados.-sin-anexos-sin-casos.pdfy, en la misma línea se expresa el colectivo Yo sí Sanidad Universal en prensa: http://www.publico.es/actualidad/534073/yosi-sanidad-universal-denuncia-que-madrid-sigue-cobrando-a-inmigrantes-en-urgencias ${ }^{10}$ Instrucció $10 / 2012$ d'accés a l'assistència sanitària de cobertura pública del CatSalut als ciutadans estrangers empadronats a Catalunya que no tenen la condició d'assegurats o beneficiaris del Sistema Nacional de Salut. Disponible en: http://www.comg.cat/uploads2/docs/fitxers/instruccio_10_2012.pdf 
empadronadas ${ }^{11}$ entre tres meses y un año y con ingresos inferiores a la renta mínima de inserción. Esta cobertura da derecho, además de la atención de urgencia, a la atención primaria y a una cobertura farmacéutica de un $40 \%$. Este nivel de atención incluye únicamente la atención especializada ofrecida por profesionales de atención primaria, así como las consultas externas de los hospitales. Para aquellas personas que pueden acreditar un empadronamiento de más de un año $\mathrm{y}$, de la misma manera, cuenten con ingresos inferiores a la renta mínima de inserción, se les posibilita el acceso también a la atención especializada. Cabe destacar que esta atención tiene una validez de un año, transcurrido este tiempo, se debe demostrar que se continúan cumpliendo los requisitos y gestionar de nuevo la solicitud de la tarjeta.

Según se indica en el informe de Médicos del Mundo (2014), el Parlamento de Cataluña aprobó una moción para que determinados grupos de población en situación administrativa irregular pudieran tener acceso a ciertos tratamientos, como la atención a las drogodependencias, prevención y atención del VIH/sida, prevención de infecciones de transmisión sexual, vacunaciones, así como aquellas dirigidas a prevenir y controlar las enfermedades transmisibles, entre otras. Pero según denuncian ONG y plataformas de profesionales, las vías administrativas para acceder a estos programas no están claras, a pesar de que sí existen en los circuitos especializados del sistema de salud público (Yo sí Sanidad Universal, 2013).Además, según plantean diversas organizaciones ciudadanas y profesionales, en Cataluña se dan irregularidades en la atención a personas inmigrantes, ya que se está facturando a mujeres embarazadas y a menores de edad no empadronadas o que llevan menos de tres meses de empadronamiento. En el resto de centros sanitarios, concretamente en aquellos concertados no gestionados por el Institut Català de la Salut, también se ha negado la atención a embarazadas y menores, o se ha procedido a su facturación y cobro ${ }^{12}$.

Por tanto, como vemos, la aprobación de este RDL 16/2012 y su implementación posterior no se está haciendo de forma homogénea y, sobretodo, no se garantiza el acceso al derecho a la salud de las personas inmigradas, ni siquiera de aquellas personas que están incluidas en la cobertura del RDL 16/2012.

\footnotetext{
${ }^{11} \mathrm{El}$ empadronamiento es posible para todas las personas independientemente de su situación administrativa, pero según denuncian diferentes organizaciones, la Administración en algunos casos puede crear entramados administrativos que lo dificultan.

${ }^{12}$ Podemos encontrar diversos ejemplos en prensa: http://ccaa.elpais.com/ccaa/2014/11/16/catalunya/1416164138 302111.html; http://www.eldiario.es/sociedad/Menores-sanidad_0_289071343.html; http://www.amnistiacatalunya.org/paginas/noticies/noticies/articulo/es-mante-lexclusiosanitaria-de-les-persones-immigrants-en-situacio-irregular-sense-atendre-le/
} 


\section{IMPLICACIONES DE LA REFORMA SANITARIA PARALAS MUJERES}

A continuación, trataremos de describir cuáles son las implicaciones que la reforma sanitaria tiene de forma específica sobre las mujeres, abordando los efectos de esta regulación sobre la prevención, detección y atención de situaciones de violencia de género, así como sus implicaciones sobre los derechos sexuales y reproductivos de las mujeres, concretamente en el acceso a la interrupción voluntaria del embarazo (IVE).

Independientemente de la regulación existente en materia de acceso al derecho a la salud, diversos estudios han puesto de manifiesto ciertas barreras a la incorporación normalizada de las mujeres inmigradas a los servicios sanitarios, al margen de su situación administrativa (Grande, Linares, Álvarez, Pastor, 2011:46). Entre éstas, destacan las que tienen que ver con aspectos culturales y religiosos; la diferente concepción de la salud y la enfermedad; la persistencia de actitudes racistas y xenófobas o prejuicios y estereotipos asumidos por parte de determinado personal sanitario; problemas de comunicación y lingüísticos; barreras administrativas y normativas; actitudes compasivas y caritativas que derivan en paternalismo; el desconocimiento por parte de las mujeres inmigrantes de nuestro sistema de salud y de los derechos y prestaciones asociados a los servicios sanitarios; y la marginación en la que muchas de las personas viven.

De todo ello, estas autoras concluían ya, que "la mujer inmigrante, aún si está regularizada y tiene derecho a una cobertura sanitaria, se encuentra con una serie de limitaciones que no existen para la población autóctona y para las que todavia no se han encontrado soluciones globales y estructurales" (2011: 48-49). Como veremos, estas limitaciones tenderán a agravarse en el marco de la política de exclusión sanitaria que consolida el RDL 16/2012. En este sentido, no debemos obviar en nuestro análisis el concepto de interseccionalidad (Crenshaw, 1993) que nos recuerda que la confluencia simultánea de diferentes variables como el género, la etnia y la pobreza, entre otros factores que afectan a las mujeres migrantes de forma especial, configura situaciones particulares de discriminación que deben considerarse de forma específica ${ }^{13}$.

\footnotetext{
${ }^{13}$ Aunque la naturaleza global de este artículo no permite un análisis en profundidad de cómo los diversos aspectos de la opresión interseccionan en las mujeres inmigradas, invitamos a leer el artículo desde esta perspectiva interseccional que las autoras consideran el enfoque adecuado para abordar la realidad de las mujeres migradas y sus situaciones de vida.
} 


\subsection{Las implicaciones de la reforma sanitaria para la prevención y detección de la violencia de género.}

La violencia de género es todavía hoy "en la mayoría de los casos, una realidad oculta y silenciada" (Estrategia Nacional para la Erradicación de la violencia contra la mujer 2013-2016), que afecta en mayor medida a ciertos segmentos de la población como es el caso de las mujeres inmigrantes. Así, diferentes autoras, organismos e instituciones públicas coinciden en afirmar la mayor vulnerabilidad ${ }^{14}$ que este grupo tiene ante las situaciones de violencia de género $^{15}$, circunstancia que se agrava en el caso de las mujeres extranjeras en situación administrativa irregular, tal y como se recoge para el caso de Cataluña en el Programa de Intervención Integral sobre la Violencia machista 2012-2015 ${ }^{16}$. Otro elemento, de gran relevancia para el tema que nos ocupa, es la percepción de las mujeres migradas sobre las instituciones públicas, ya que son vistas a menudo como una amenaza, más que como una fuente de protección, que se materializa en el miedo a ser expulsadas si, al encontrarse en situación administrativa irregular, acuden a poner una denuncia; en la sensación de culpa si la pareja puede ser expulsada o ellas separadas de sus hijas e hijos; o en el miedo a no ser creídas o a que su denuncia pueda tener efectos en su proceso de regularización.

Esta mayor exposición ante situaciones de violencia machista queda refrendada en los datos de la Macroencuesta de Violencia de género 2011, que se recogen en el texto de la Estrategia Nacional para la Erradicación de la violencia contra la mujer (2013-2016) ${ }^{17}$. Además de la cifra de 202 mujeres extranjeras fallecidas a manos de sus parejas o exparejas durante el periodo comprendido entre 2003 y 2012 , que supone el $30,8 \%$ del total de víctimas con nacionalidad conocida, el estudio indica que las mujeres extranjeras declaran haber sufrido alguna vez en la

\footnotetext{
${ }^{14}$ Siguiendo la guía Claves contextuales para la detección de la violencia de género en mujeres inmigradas desde los servicios de Atención Primaria de la Fundación Ceimigra más que referirnos a la vulnerabilidad de las personas, es conveniente tener presente que son las circunstancias y condiciones de vida lo que provoca como consecuencia esa vulnerabilidad. Por tanto, "más que de personas vulnerables, lo más preciso sería hablar de sistemas vulnerabilizadores y de experiencias de vulnerabilización”. Este será, en consecuencia, el significado que le daremos a este concepto a lo largo de los siguientes apartados.

${ }^{15}$ Merchán, 2013; Federación de Mujeres Progresistas, 2007; Amnistía Internacional (2003); Estrategia Nacional para la Erradicación de la Violencia contra la mujer (2013-2016); Plan de Atención y Prevención de la Violencia de Género en Población Extranjera Inmigrante (2009-2012); Fundación CEIMIGRA, 2014.

${ }^{16} \mathrm{http}: / /$ dones.gencat.cat/web/.content/04 temes/docs/piivm.pdf

${ }^{17}$ http://www.um.es/estructura/unidades/u-igualdad/intranet/docs/estratgnacion.pdf
} 
vida situaciones de violencia de género en mayor proporción que el total de las encuestadas ${ }^{18}$.

En el caso de Cataluña, la Encuesta de violencia machista de 2010 indica que el nivel de exposición a la violencia de género es inequívocamente superior entre las mujeres nacidas fuera del Estado español y que la prevalencia en las mujeres extranjeras es aproximadamente el doble que en las mujeres catalanas ${ }^{19}$.

En el artículo Les víctimes mortals per violència masclista en l'àmbit de la parella. Anàlisi de casos a Catalunya del 2008 al 2012(Alfageme,Casella, Guiu, Llavari,2013) en el que se analizan los datos recogidos por la policía de la Generalitat- Mossosd'Esquadra del 2008 al 2012, se indica que el 45\% de las mujeres asesinadas por violencia machista en el ámbito de la pareja son de nacionalidad extranjera. Por áreas geográficas, el $4 \%$ procedían de la UE; el 7\% del resto de Europa; el 2\% de África subsahariana; el 14\% de América del Sur y Central; el 13\% del Magreb; y el 5\% de países asiáticos. Teniendo en cuenta que la población extranjera en Cataluña representaba aproximadamente en 2011 el 16\% del total, los datos muestran una sobrerrepresentación de este grupo demográfico entre las mujeres asesinadas por violencia machista.

Por último, respecto a la evolución de las órdenes de protección en el periodo, según datos del Observatorio contra la violencia doméstica y de género del Consejo General del Poder Judicial, en el caso de Cataluña se concedieron 8.849 órdenes de protección para mujeres extranjeras en el cuatrienio 2010-2013, cifra que representa el $38 \%$ del total de órdenes concedidas.

Como mencionábamos anteriormente, la situación de irregularidad administrativa tiende a agravar las posibilidades de prevención e identificación de víctimas de violencia de género. En este sentido, y a pesar de las modificaciones normativas en los últimos años con el fin de garantizar y ampliar el marco para la prevención y lucha contra la violencia de género de este grupo de población, algunos datos nos permiten observar hacia dónde está evolucionando el fenómeno, fruto también del endurecimiento de la política migratoria del Estado español en los

\footnotetext{
${ }^{18} \mathrm{El} 14,5 \%$ de las mujeres que manifestaron haber sufrido maltrato en el último año son mujeres extranjeras; el $20,9 \%$ de las mujeres extranjeras encuestadas habían sufrido violencia de género alguna vez en la vida, frente al 10,9\% del total de mujeres encuestadas; y el 5,8\% lo estaban sufriendo en el último año, frente al $3 \%$ del total de mujeres encuestadas.

${ }^{19}$ http://victimesviolencia.gencat.cat/ca/03 materials i dades_sobre violencia masclista i domestica/02 documentacio sobre violencia masclista i domestica per a professionals/0 5 resultats de lenquesta de violencia masclista/
} 
últimos años. La potenciación de las medidas de identificación de población extranjera en situación de irregularidad administrativa, detención en Centros de Internamiento de Extranjeros y posterior expulsión, en el caso de que esta pueda darse, tiende a agravar este recelo hacia las instituciones del Estado, incluidos los servicios sanitarios.

Así, en el artículo La denúncia i el silenci: dues estratègies de les dones per lluitar contra la violència masclista (Bodelón, 2013: 128), se identifica el marco normativo de extranjería como una de las barreras para la protección de las mujeres migrantes en situación irregular víctimas de violencia de género. La autora indica que " a pesar de las reformas recientes, la Ley de extranjería actual establece la apertura de expediente de expulsión a las mujeres migrantes en situación irregular que, tras denunciar por violencia de género, no logren acreditar los hechos en el proceso penal". Los datos de denuncias archivadas y sentencias absolutorias (alrededor de la mitad en cada caso), señaladas por la autora, continúan generando miedo y desconfianza entre las mujeres.

En el contexto de la lucha contra la violencia de género, sin embargo, los servicios sanitarios cumplen un papel fundamental a la hora de detectar mujeres en posibles situaciones de violencia y, dentro de estos, la atención primaria tiene un rol esencial. Según datos del Ministerio de igualdad del 2011, más del $60 \%$ de los casos de violencia de género que se detectaban en el ámbito del Sistema Nacional de Salud eran descubiertos en este escalón asistencial. Por su parte, Médicos del Mundo (2014) añade a estos datos que sólo una minoría de mujeres que han sufrido violencia de género aporta parte de lesiones al realizar una denuncia: en 2012, de las 128.500 denuncias, apenas un $11 \%$ presentaba parte de lesiones.

La encuesta elaborada por la European Union Agency for Fundamental Rights (FRA) ${ }^{20}$, muestra que las mujeres en situación de violencia contactan con personal sanitario con más frecuencia que con la policía u otros servicios, por lo que en el informe se afirma que los profesionales de la salud pueden jugar un papel destacado en la identificación y canalización de los casos de violencia contra las mujeres. En el caso de España las diferencias son menores que a nivel europeo pero el patrón es el mismo, pues se contacta en mayor medida con personal sanitario que

\footnotetext{
${ }^{20}$ Los resultados de la encuesta de la FRA sobre la violencia contra la mujer se basan en entrevistas personales realizadas a 42.000 mujeres en los 28 Estados miembros de la UE, con una media de 1.500 entrevistas por país. Los resultados son representativos de las experiencias y las opiniones de mujeres con edades entre los 18 y los 74 años residentes en la UE.

http://www.msssi.gob.es/ssi/violenciaGenero/laDelegacionInforma/pdfs/F_R_A.pdf
} 
con la policía ( $26 \%$ contacta con un hospital o centro de salud frente a $20 \%$ que contacta con la policía).

El rol que los servicios sanitarios cumplen en este ámbito adquiere un sentido más evidente en aquellos casos, como en el de las mujeres migrantes, en los que su situación de vulnerabilidad condicionada por los factores anteriormente mencionados dificulta su acceso a los instrumentos jurídicos previstos, pero que en cambio sí acceden en ocasiones a los servicios sanitarios, según reconoce el Protocolo común para la actuación sanitaria ante la violencia de género (2012). Así, el propio documento recuerda que en el grupo de las mujeres migrantes en situación administrativa irregular o con barreras idiomáticas, las dificultades para la detección de casos de violencia de género son más elevadas. A ello, este protocolo añade que:

"Desde los servicios sanitarios se puede desempeñar un papel crucial para ayudar a las mujeres que sufren violencia de género, ya que la mayoría de las mujeres entran en contacto con ellos en algún momento de su vida (consultas a demanda y preventivas, embarazo, parto, cuidado médico de los hijos o hijas, cuidado de las personas mayores, etc.). Además, las mujeres que sufren cualquier forma de violencia de género acudirán con mayor frecuencia a los servicios sanitarios, en particular a Atención Primaria, Urgencias, Obstetricia y Ginecología y Salud Mental." (Institut Català de les Dones, 2012: 35-36)

De forma similar, el documento Claves contextuales para la detección de violencia de género en mujeres inmigradas desde los servicios de Atención Primaria de la Fundación Ceimigra (2014) recoge la necesidad de establecer una relación de confianza con las mujeres inmigradas como clave fundamental en el proceso de detección precoz de casos de violencia de género. En este proceso, el tiempo se convierte en una variable esencial que normalmente es más dilatada en el caso de las mujeres inmigradas como resultado de factores culturales, entre otros.

De todo lo anterior, se concluye que la presencia de situaciones de violencia de género en la población inmigrante alcanza cifras significativas y que, a pesar de las dificultades y barreras que en algunos casos las mujeres migrantes tienen para acceder a los servicios sanitarios, estos continúan teniendo un rol de gran relevancia en la detección de casos de violencia machista. Por tanto, si estas mujeres, según el RDL 16/2012, ven restringido su acceso regular a los servicios de atención primaria, la influencia de este hecho sobre la posibilidad de detectar casos de violencia de género se limita significativamente.

Médicos del Mundo en su informe de 2014, en el que hace balance de la aplicación dela nueva normativa, menciona de forma explícita lo siguiente: 
"No puede dejar de señalarse otro (efecto)que es especialmente sangrante: la pérdida de oportunidades de que en el sistema sanitario se detecten casos de violencia de género. La exclusión del sistema sanitario de mujeres inmigrantes sin permiso de residencia impide que se beneficien de la situación privilegiada para la detección de maltrato que se produce dentro de los centros sanitarios." (Médicos del Mundo, 2014:7)

En idéntica línea, el informe REDES Registro Estatal para la Denuncia de la Exclusión Sanitaria, publicado en septiembre de 2014 por Yo sí Sanidad Universal y la Sociedad Madrileña de Medicina de Familia y Comunitaria (SoMaMFYC) recoge el intento de emisión de facturas a víctimas de violencia de género después de acudir a los servicios sanitarios para realizar un parte de lesiones ${ }^{21}$.

La situación es igualmente grave para aquellos grupos de mujeres migrantes en situación irregular que sufren situaciones de extrema vulneración de derechos, como las mujeres tratadas con fines de explotación sexual o lasque han sufrido violencia sexual. Respecto a las primeras, y aunque la nueva regulación contempla estos casos como una de las excepciones para el derecho a la asistencia sanitaria, esto sólo ocurrirá si reciben autorización de residencia temporal por estar acogidas al periodo de restablecimiento y reflexión ${ }^{22}$. Este requisito, además de restrictivo, es inaplicable en un número muy elevado de casos. Según las cifras citadas por la oficina del Defensor del Pueblo, en el año 2009 fueron detectadas 6.157 personas en situación de riesgo; en 2010 se identificaron 15.075; y en 2011, 14.370. Por su parte, los datos de la Fiscalía General del Estado relativos a 2012 indican que se ofreció el periodo de reflexión a 149 mujeres que eran presuntas víctimas de trata con fines de explotación sexual (Médicos del Mundo, 2014:18).

En el caso de Cataluña, existe un Protocolo de Protección de las Víctimas de Tráfico de Seres Humanos en Cataluña desde octubre de 2013, que adapta el protocolo marco estatal.

A su vez, las medidas contempladas en la Estrategia Nacional para la Erradicación de la Violencia contra la Mujer (2013-2016), como la incorporación

\footnotetext{
${ }^{21}$ Informe accesible en http://yosisanidaduniversal.net/noticias.php/el-primer-informe-redesconstata

${ }^{22}$ El periodo de restablecimiento y reflexión no se expide ni se concede a todas las víctimas de trata; sólo se aplica a víctimas y (presuntas víctimas) de trata de personas, que están en situación administrativa irregular en España, en el caso de que necesiten tomarse un tiempo para decidir si quieren o no colaborar con las autoridades en la persecución del delito.
} 
de este grupo de mujeres al Protocolo común para la actuación sanitaria ante la violencia de género (2012), entre otras medidas, no tendrán tampoco impacto en virtud de la aplicación del RDL 16/2012.

En el caso de las víctimas de violencia sexual, el estudio Violadas y expulsadas de la Fundación Aspacia (2013) identifica la reciente reforma de acceso a la atención sanitaria como un ejemplo de regulación que supone una discriminación directa de las mujeres migrantes en situación irregular frente a la violencia sexual, ya que considera que "impide u obstaculiza a mujeres víctimas de violencia sexual en situación irregular, el acceso a tratamientos fundamentales para su recuperación" (2013:21).

Por tanto, y volviendo al estudio de Médicos del Mundo, la ONG advierte que "limitar a los servicios de urgencias la posibilidad de detección significa un importante retroceso en la estrategia para reducir esta forma de violación contra los derechos humanos de las mujeres" (2014:18). La excepción, como comentábamos anteriormente $\mathrm{y}$, según informa la entidad, la constituyen las comunidades de Andalucía y Asturias donde las mujeres inmigrantes en situación administrativa irregular tienen derecho al acceso al sistema de salud normalizado en iguales condiciones que el resto. Sin embargo, la desconfianza y los temores a la hora de acercarse a estos servicios en el nuevo contexto pueden limitar la eficacia de las medidas.

En conclusión, la aplicación del RDL 16/2012 limita las posibilidades de prevenir la violencia de género al eliminar una de las vías de detección fundamentales como son los servicios de atención primaria, a lo que se une el incremento de las barreras y recelos existentes por parte de la población inmigrante, agravados en el caso de quienes están en situación de irregularidad administrativa. Las consecuencias de este hecho serán mucho más acusadas en aquellos casos de especial gravedad como el de las mujeres tratadas con fines de explotación sexual o aquellas que han sufrido violencia sexual.

\subsection{Las implicaciones de la reforma sanitaria para los derechos sexuales y reproductivos de las mujeres inmigradas.}

El ejercicio de los derechos sexuales y reproductivos requiere un contexto en el que todas las personas puedan decidir libremente sobre temas relacionados con su sexualidad y que, entre otras libertades, exista el acceso a información, educación y servicios de salud sexual y reproductiva, lo que el RDL 16/2012 imposibilita, especialmente, en el caso de las mujeres inmigradas en situación administrativa irregular. 
A continuación, nos centraremos concretamente en las implicaciones de la reforma sanitaria sobre la interrupción voluntaria del embarazo. Tal y como apunta el Comité para la Eliminación de la Discriminación contra las Mujeres de Naciones Unidas (CEDAW), la negación o el rechazo de acceso a procedimientos médicos que sólo las mujeres requieren, como es el caso del aborto, constituyen una discriminación de género.

En la actualidad en el Estado español continua existiendo un serio y constante cuestionamiento ${ }^{23}$ sobre el derecho a la interrupción voluntaria del embarazo, recogido en la Ley $2 / 2010$ de salud sexual y reproductiva y de la interrupción voluntaria del embarazo $^{24}$, y que atenta contra la autonomía reproductiva de las mujeres, uno de los derechos reconocidos en laCarta de Derechos Sexuales y Reproductivos de la International Planned Parenthood Federation (IPPF).Si a esta situación de constante inseguridad y amenaza al derecho a decidir de las mujeres sobre su salud sexual y reproductiva, sumamos las dificultades añadidas que ya tienen las mujeres inmigradas en situación irregular para acceder a los servicios públicos de salud sexual y reproductiva, la situación se agrava todavía más. Tal y como señala Médicos del Mundo en su informe:

"Las mujeres inmigrantes sin tarjeta sanitaria que desean interrumpir su embarazo aún dentro del marco legal regulado por Ley Orgánica de salud sexual y reproductiva y de la interrupción voluntaria del embarazo 2/2010 encuentran grandes barreras para ser atendidas en los servicios sanitarios"(2014:19).De la misma manera "la exclusión del sistema público de salud en los niveles de Atención Primaria y Especializada, impide el acceso a programas de información y asesoramiento en métodos anticonceptivos"(2014:18).

Por tanto, ni se facilitan los medios y la información necesaria para prevenir los embarazos no deseados, ni se garantiza la libertad para decidir sobre su capacidad reproductiva, de forma quelas mujeres en situación administrativa irregular encuentran vulnerados sus derechos sexuales y reproductivos.

23En este sentido, durante la actual legislatura, el gobierno ha presentado el anteproyecto de Ley Orgánica para la protección de la vida del concebido y de los derechos de la mujer embarazada (diciembre de 2013), que finalmente fue retirado el 23 de septiembre de 2014. Asimismo, en la actualidad se encuentra en debate la propuesta de modificación del derecho a la interrupción voluntaria del embarazo sin consentimiento paterno para las menores de 18 años.

${ }^{24}$ Ley Orgánica $2 / 2010$, de 3 de marzo, de salud sexual y reproductiva y de la interrupción voluntaria del embarazohttp://www.boe.es/diario_boe/txt.php?id=BOE-A-2010-3514. 
Tal y como apunta el informe Anàlisi de l'impacte de la immigració en les interrupcions voluntàries de l'embaràs a Catalunya ${ }^{25}$, las mujeres inmigradas son un grupo muy heterogéneo; y tal como muestran diversos estudios, el origen de las mujeres, ya sea autóctona o inmigrada, no es en sí mismo un factor de riesgo para la interrupción voluntaria del embarazo, ya que más allá de las características culturales, se han de tener en cuenta otras circunstancias que inteseccionan y determinan las diferencias, como son, por ejemplo, el acceso a los servicios de salud sexual y reproductiva, el idioma, la situación económica, la situación laboral y su situación administrativa. No se trata, por tanto, de la suma de desigualdades, sino de que cada una de ellas intersecciona de forma diferente en cada situación personal y grupo social mostrando estructuras de poder existentes en el seno de la sociedad (Expósito, 2012: 210), por tanto, también en el caso de las mujeres inmigradas.

En nuestro contexto, y siguiendo el informe Acceso al aborto en el Estado español. Un mapa de inequidad ${ }^{26}$, las mujeres procedentes de América, Europa del Este y África consideran las dificultades económicas para criar una hija o un hijo el principal motivo para interrumpir el embarazo. En cambio, las mujeres asiáticas lo atribuyen simplemente al deseo de no tener más descendencia. Respecto al método contraceptivo más usado por las mujeres inmigradas, se trata del preservativo, pero la falta de anticoncepción es importante y más frecuente en las mujeres asiáticas, que según el estudio, utilizan en mayor número de casos los llamados métodos naturales ${ }^{27}$. También las mujeres provenientes de Europa del Este utilizan estos métodos y son asimismo las que presentan un número mayor de IVE de repetición. Como característica común a las mujeres entrevistadas para el estudio, la anticoncepción postcoital es poco conocida, a excepción de las mujeres latinoamericanas. Por tanto, es importante romper con los estereotipos frecuentemente asociados a las mujeres inmigradas en cuanto a su salud sexual y reproductiva, así como tener en cuenta cuáles son las necesidades que deben cubrirse para poder disfrutar plenamente de sus derechos sexuales y reproductivos.

\footnotetext{
${ }^{25}$ SERVEI D’INFORMACIÓ I ESTUDIS (2011): Anàlisi de l'impacte de la immigració en les interrupcionsvoluntàries de l'embaràs a Catalunya. Barcelona: Departament de Salut. Generalitat de Catalunya.www20.gencat.cat/docs/salut/Home/El\%20Departament/Estadistiques \%20sanitar ies/Dades $\% 20 \mathrm{de} \% 20$ salut $\% 20 \mathrm{i} \% 20$ serveis $\% 20$ sanitaris/Interrupcio $\% 20$ voluntaria $\% 20 \mathrm{de} \% 2$ 0lembaras $\% 20(\mathrm{IVE}) /$ documents/immigracio ives.pdf

${ }^{26}$ Estudio realizado por el Grupo de interés Español en Población, Desarrollo y Salud Reproductiva, y la Asociación de Clínicas Acreditadas para la Interrupción de Embarazo (ACAI).

${ }^{27}$ Los métodos denominados naturales son el método del calendario o Ogino-Knaus, la temperatura basal y el método del moco cervical o Billings.
} 
En esta misma línea, y según el estudio Características sociodemográficas de las mujeres que realizan una interrupción de la gestación en España ${ }^{28}$, a pesar de que el $80 \%$ de las mujeres extranjeras que participaron en él aseguraban tener información sobre los métodos anticonceptivos, más del 70\% los aplicaron mal, de lo que puede deducirse que no existe un acceso real o sencillo a los centros o profesionales que se encargan de la orientación contraceptiva. Otro factor de especial interés que se señala en este estudio, se refiere al tiempo de espera y la inaccesibilidad profesional para aclarar dudas, realizar seguimientos del método, etc. Por todos estos motivos, es imprescindible garantizar el acceso de las mujeres inmigradas en situación irregular a los servicios públicos de salud sexual y reproductiva.

Si nos fijamos ahora en las estadísticas en relación a las IVE en Cataluña, los últimos datos disponibles que desagregan la población según el país de procedencia, son los correspondientes al $2012^{29}$. Ese año se realizaron un total de $21.950 \mathrm{IVE}$, y las cifras mostraron un ligero descenso de estas entre mujeres de origen extranjero, con un $40,5 \%$ del total, mientras que el porcentaje se incrementaba entre las mujeres autóctonas. Los países de procedencia más frecuentes son Bolivia, Marruecos, Ecuador y Rumanía y, del total de interrupciones realizadas, el $52,8 \%$ han tenido lugar en un centro público. Si nos fijamos en las edades de las mujeres extranjeras que interrumpen su embarazo en Cataluña, el grupo más numeroso se encuentra entre los 20 y los 24 años, con un $35,77 \%$ de los casos, seguida de la franja de edad de los 15 a los 19 años con un $27,92 \%$ y del grupo de edad comprendido entre los 25 y los 29 con un 27,58\% de total de las IVE. Por eso, es fundamental proteger el acceso a los recursos públicos de atención y educación en salud sexual y reproductiva de todas las mujeres, especialmente de la población más joven.

Teniendo en cuenta los datos anteriores, la exclusión de las mujeres inmigrantes en situación irregular en virtud del RDL 16/2012de acceso a la interrupción voluntaria del embarazo en el sistema desanidad pública genera, y generará, una situación de clara desigualdad entre estas, ya que no todas tienen la misma capacidad económica para hacer frente a una IVE fuera de la sanidad

\footnotetext{
${ }^{28}$ ACAI (2010): Características sociodemográficas de las mujeres que realizan una interrupción de la gestación en España. Disponible en: http://www.acaive.com/pdf/Caracteristicas\%20Sociologicas\%20mujeres-AbortoAnticoncepcion $\% 20$ fallida $\% 20 \mathrm{y} \% 20$ Aborto $\% 20$ Provocado.pdf

${ }^{29} \mathrm{La}$ interrupcióvoluntària de l'embaràs a Catalunya. 2012. Disponible en: http://www20.gencat.cat/docs/canalsalut/Minisite/ObservatoriSalut/ossc_Dades estadistique s/Estat_salut estils_vida/Temes_especifics_salut/Salut_sexual_reproductiva/Fitxers estatics Interrupcio_voluntaria_embaras_2012.pdf
} 
pública. De esta manera, la única opción disponible para estas mujeres sería interrumpir el embarazo de forma clandestina e insegura.

Por último, no queremos dejar de evidenciar que la violencia de género tiene claros puntos de intersección con la salud sexual y reproductiva. Entre las mujeres en situación de violencia son comunes las dificultades para negociar la planificación familiar y el uso irregular de los métodos anticonceptivos. Por otro lado, las mujeres en situación de violencia en la pareja se ven sometidas a limitaciones en el proceso de decisión sobre una IVE, limitación que se derivan principalmente del aislamiento que conlleva la propia situación de violencia. Por tanto, cualquier limitación legal a la interrupción voluntaria del embarazo tendrá un impacto específico sobre las mujeres en situación de violencia.

En conclusión, la aplicación del RDL 16/2012 conlleva un recorte de los derechos sexuales y reproductivos de las mujeres inmigradas en situación administrativa irregular en Cataluña y en el Estado español y sobre el derecho a decidir sobre su cuerpo y su capacidad reproductiva.

\section{LAS ESTRATEGIAS DE LA SOCIEDAD CIVIL EN LA LUCHA CONTRA LA APLICACIÓN DEL RDL 16/2012.}

Desde el inicio de la implementación del RDL 16/2012 son muchas las entidades, colectivos y grupos de profesionales que se han organizado con el objetivo de, en primer lugar, defender el modelo de sanidad pública universal para todas las personas, independientemente de su nacionalidad, situación administrativa, laboral o cualquier otra condición excluyente y, en segundo lugar, garantizar la atención a la salud a las personas actualmente excluidas a causa de la implementación del RDL 16/2012.

Cada una de las organizaciones desarrolla sus actividades en diferentes líneas, como por ejemplo, sensibilizar a la población general y movilizarla para protestar con el fin de retirar el RDL 16/2012; otras organizaciones y colectivos se centran en informar a las personas afectadas sobre cómo obtener la tarjeta sanitaria, así como, en colaboración con profesionales y otras entidades, encontrar la vía para garantizar la asistencia sanitaria a las personas que ya no tienen cobertura ni posibilidad de conseguir la tarjeta sanitaria. Otra línea de trabajo es la de información, a través de la difusión de las consecuencias de la aplicación del RDL 16/2012 en los medios de comunicación y las redes sociales, así como la sensibilización entre la comunidad médica, con el objetivo de que el personal médico conozca y ejerza su derecho a la desobediencia profesional y objeción de conciencia. Por último, es de vital importancia la incidencia política, a través del diálogo con las diversas administraciones y sus representantes, para exigir 
soluciones a los problemas derivados de la aplicación de la normativa y solicitar la retirada del RDL 16/2012.

En Cataluña, destaca la labor de diversas organizaciones, como por ejemplo Yo sí Sanidad Universal que, entre otras actividades, organizan grupos autogestionados de personas voluntarias en diversos barrios, que acompañan a quienes necesitan asistencia sanitaria y no tienen cobertura. Si estas personas no son atendidas en la red pública, se busca la atención sanitaria a través de profesionales objetores, o algún servicio paralelo, como por ejemplo, el Espacio del inmigrante, que una vez a la semana atiende a personas sin cobertura sanitaria en el barrio del Raval de Barcelona. Además, la Plataforma per una Atenció Sanitària Universal a Catalunya (PASU_cat)reúne a 36 entidades y profesionales de la salud, entre las que se encuentran organizaciones como el Comité 1er de Desembre, que trabaja en el ámbito del VIH/sida, SOS Racisme, l'ONGDMetges del Món, la Xarxa de Dones per la Salut, una red de asociaciones y mujeres feministas motivadas por la salud de las mujeres, formada, a su vez, por 21 asociaciones, o la PARS la Plataforma de Afectados por los Recortes Sanitarios. Sin el trabajo que realizan todas estas organizaciones y profesionales, las consecuencias del RDL 16/2012 hubieran sido más devastadoras todavía.

\section{CONCLUSIONES}

Es imprescindible tener en cuenta que la exclusión que ha generado el RDL 16/2012 no es sólo un problema individual, que hasta el momento ya ha causado varias muertes, sino que supone un cambio de modelo sanitario que afecta a la sociedad en su conjunto, y provoca una pérdida de calidad en la atención sanitaria que repercute en la salud de toda la ciudadanía del Estado español.

No obstante, la reforma sanitaria afecta especialmente a los grupos de población que se encuentran en situación de vulnerabilidad, entre los que destacan la población inmigrante en situación de irregularidad administrativa, donde las mujeres ocupan un lugar relevante. En un contexto de restricción y retroceso de los derechos de las mujeres y, concretamente, de constante amenaza de los derechos sexuales y reproductivos que afecta al conjunto de la sociedad española, una reforma como la implementada hace ya dos años no conduce más que a un empeoramiento de la situación. Por otro lado, y de forma específica, se está produciendo también una vulneración de derechos fundamentales como la prevención, detección y protección en materia de violencia de género con serias consecuencias para las vidas de las mujeres.

La nueva legislación, lejos de suponer un ahorro en términos económicos, implica, por el contrario, repercusiones negativas para su salud física, la 
vulneración del derecho humano básico de acceso a la salud, consagrado en la legislación estatal, así como en diversos pactos y acuerdos de carácter internacional ratificados por el Estado español. En resumen, la aplicación del RDL/16/2012 nos lleva a una segmentación todavía mayor de la sociedad y a nuevas exclusiones, basadas en la situación laboral de las personas, su situación administrativa y capacidad económica.

La sociedad civil y profesionales sanitarios se han organizado con el objetivo de visibilizar las consecuencias de este RDL 16/2012, denunciar el cambio de modelo sanitario, conseguir volver a la universalidad del sistema sanitario y apoyar, mediante una labor de información, asesoría y acompañamiento, a los grupos de población que han quedado excluidos de la atención sanitaria. Cabe recordar que medidas anteriormente implementadas, como la del copago sanitario, han sido retiradas gracias a la actividad de denuncia de la sociedad en un ejercicio que ha supuesto un reflejo de lo que esta puede lograr en la defensa de los derechos del conjunto de la población.

Es imprescindible más investigación feminista que explore los efectos del RDL 16/2012 sobre las mujeres en temas de vital importancia como la violencia machista y el derecho a decidir sobre sus cuerpos. En este sentido, resultaría también de gran interés analizar con una mayor profundidad la incoherencia entre distintas políticas como las de extranjería, violencia de género, trata, sanidad, igualdad de género, entre otras. Tal y como se ha ejemplificado a lo largo del artículo, mientras nos encontramos con iniciativas políticas que tratan de proteger específicamente a determinados grupos en situación de vulnerabilidad, vemos que al mismo tiempo otras restringen el ejercicio de derechos asociados con las situaciones protegidas anteriormente. Asimismo, será necesario acompañar a las entidades y profesionales en su labor de denuncia y difusión del impacto de esta reforma sanitaria sin precedentes en el Estado español.

\section{REFERENCIAS BIBLIOGRÁFICAS Y NORMATIVAS}

ALFAGEME, ALBA.; CASELLA, MIREIA., GUIU CRISTINA. y LLAVARI, MARÍA(2013);Les víctimesmortals per violènciamasclista en l'àmbit de la parella. Anàlisi de casos a Catalunya del 2008 al 2012. Apunts de seguretat. Violènciamasclista $i$ domèstica. Polítiques $i$ actuacions, $\mathrm{n}^{\circ}$ 12, noviembre 2013, pp. 183-2015

AMNISTİA INTERNACIONAL (2013):El laberinto de la exclusión sanitaria. Vulneraciones del derecho a la salud en las Islas Baleares. Disponible en: 
https://doc.es.amnesty.org/cgi-

bin/ai/BRSCGI?CMD $=$ VERDOC\&BASE $=$ SIAI\&SORT $=-$ FPUB $\& D O C R=1 \& R N G=10 \& S E P A R A D O R=\& \& I N A I=E U R 4160013[$ Fecha de acceso: 08 de julio de 2014]

AMNISTÍA INTERNACIONAL (2003):Mujeres invisibles, abusos impunes. Mujeres migrantes indocumentadas en España ante la violencia de género en el ámbito familiar. Disponible en:

http://www.redfeminista.org/nueva/uploads/Amnistia\%20Definitivo\%20 1de\%20julio. pdf[Fecha de acceso: 01 de julio de 2014]

ARNOSO, MAITANE;ARNOSO, AINARA; MAZKIARAN, MIKELy IRAZU, AINHOA (2012):Mujer inmigrante y violencia de género: factores de vulnerabilidad y protección social,Migraciones 32, pp. 169-200

ASOCIACIÓN DE CLÍNICAS ACREDITADAS PARA LA IVE (2014): Condiciones que motivan el aborto provocado. Disponible en:

www.acaive.com/condiciones-que-motivan-el-aborto-provocado-2/publicaciones

[Fecha de acceso: 05 de diciembre de 2014]

BODELÓN, ENCARNA(2013): La denúncia i el silenci: duesestratègies de les dones per lluitar contra la violènciamasclista.Apunts de seguretat. Violènciamasclista $i$ domèstica. Polítiques i actuacions, $\mathrm{n}^{\circ} 12$, noviembre 2013, pp. $123-148$.

CALVO, GERMÁN; CAMACHO, RAFAELA (2014): La violencia de género: evolución, impacto y claves para su abordaje, Enfermería Global, $\mathrm{n}^{\mathrm{o}} 33$, pp. 424-439.

CONSEJO GENERAL DEL PODER JUDICIAL. Datos estadisticos del Observatorio contra la violencia doméstica y de género. Disponible en Observatorio contra la violencia doméstica y de género.

http://www.poderjudicial.es/cgpj/es/Temas/Violencia-domestica-y-degenero/Actividad-del-Observatorio/Datos-estadisticos[Fecha de acceso: 20 de junio de 2014]

CRENSHAW WILLIAMS KIMBERLE (1991): Mapping the Margins:Intersectionality, Identity Politics, and Violence Against Women of Color. Stanford Law Review 43, no. 6, pp. 1241-1299.

DEPARTAMENT DE SALUT (2013):Enquesta de salut de Catalunya 2012. Informe delsprincipals resultats. Generalitat de Catalunya. Disponible en: http://www20.gencat.cat/docs/canalsalut/Minisite/ObservatoriSalut/ossc Dades estadistiques/Estat_salut estils vida/Informacio general enquestes salut/Enq uestes salut/Fitxers estatics/Enquesta salut 2012 edicio maig.pdf [Fecha de acceso: 20 de junio de 2014]

ESTRELA, EDITE (2013):Informe sobre salud sexual y reproductiva y derechos afines (2013/2040 (INI)). Bruselas, Parlamento Europeo, Comisión de Derechos de la Mujer e Igualdad de Género.

EXPOSITO MOLINA, CARMEN (2012): ¿Qué es eso de la interseccionalidad? Aproximación al tratamiento de la diversidad desde la perspectiva de género en España. Revista de Investigaciones Feministas, vol. 3,pp. 203-222. Disponible 
en: http://revistas.ucm.es/index.php/INFE/article/view/41146 [Fecha de acceso: 20 de diciembre de 2014]

FEDERACIÓN INTERNACIONAL DE PLANIFICACIÓN FAMILIAR (2008): Carta de Derechos Sexuales y Reproductivos. Disponible en:

ippf sexual rights declaration pocket guide spanish.pdf [Fecha de acceso: 05 de diciembre de 2014]

FEDERACIÓN INTERNACIONAL DE PLANIFICACIÓN FAMILIAR (2013): Barómetro de acceso de las mujeres a métodos anticonceptivos modernos en 10 países de UE. Disponible en:

www.ginealmeria.es/pdf/BarometroAnticonceptivos.pdf [Fecha de acceso: 05 de diciembre de 2014]

GENERALITAT DE CATALUÑA: Ley 5/2008, de 24 de abril, del derecho de las mujeres a erradicar la violencia machista. Disponible en:

https://www.boe.es/diario boe/txt.php?id=BOE-A-2008-9294[Fecha de acceso: 20 de junio de 2014]

GENERALITAT DE CATALUÑA. Protocolo de protección de las víctimas de tráfico de seres humanos en Cataluña.. Disponible en:

http://premsa.gencat.cat/pres fsvp/docs/2013/10/17/11/42/02a5c5e2-dd84-4af4-b6db29bb016553e5.pdf[Fecha de acceso: 20 de junio de 2014]

GOBIERNO DE ESPAÑA (2009):Plan de atención y prevención de la violencia de género en población extranjera inmigrante (2009-2012).Disponible en

https://www.msssi.gob.es/ssi/violenciaGenero/Documentacion/medidasPlanes/DOC/Pla $\mathrm{n}$ atencion prevencion violencia_genero poblacion extranjera inmigrante $(20$ 09 2012).pdf[Fecha de acceso: 02 de julio de 2014]

GRANDE, M ${ }^{\mathrm{a}}$ LUISA; LINARES, MANUEL; ÁLVAREZ, CARMEN (2011): El abordaje de la salud sexual y reproductiva en mujeres inmigrantes desde un enfoque de

género, Cultura de los cuidados, $2^{\circ}$ cuatrimestre 2011, Año XV, N 30, pp. 45-51.

GRUPO DE INTERÉS ESPAÑOL EN POBLACIÓN, DESARROLLO Y SALUD REPRODUCTIVA y ASOCIACIÓN DE CLÍNICAS ACREDITADAS PARA LA INTERRUPCIÓN DEL EMBARAZO (2008): Acceso al aborto en el Estado español. Un mapa de inequidad. Disponible en:http://www.acaive.com/pdf/Acceso-al-aborto-en-Espana.pdf $\quad[$ Fecha de acceso: 15 de junio de 2014]

INTERNATIONAL PLANNED PARENTHOOD FEDERATION AND SEXUAL RIGHTS INITIATIVE (2012): Derechos sexuales y el examen periódico universal. Disponible en:

http://issuu.com/ippfresources/docs/universalperiodicreview toolkit 2012[Fecha acceso: 02 junio 2014]

LEMA, MARGARITA (2013): La reforma sanitaria en España: Especial referencia a la población inmigrante en situación administrativa irregular, Eunomía. Revista en Cultura de la Legalidad, $\mathrm{N}^{\circ}$ 5, septiembre 2103 - febrero 2014, pp. 95-115

LÓPEZ, RAQUEL (2013): Mujer inmigrante víctima de violencia de género, en REDUR 11, diciembre 2013, págs. 199-229. 
LÓPEZ, EVA (2007):Mujeres inmigrantes y violencia de género. Aproximación diagnóstica a tres años de la existencia de la Ley de Medidas de Protección Integral contra la Violencia de Género, Madrid: FEMP

MÉDICOS DEL MUNDO (2014): Dos años de reforma sanitaria: más vidas humanas en riesgo. Disponible en:

http://www.actasanitaria.com/wp-content/uploads/2014/04/informe-medicos-delmundo-impacto-rd.pdf[Fecha de acceso: 20 de junio de 2014]

MÉDICOS DEL MUNDO (2013):El impacto de la reforma sanitaria en el derecho a la salud. Disponible en:

http://www.medicosdelmundo.org/derechoacurar/wpcontent/uploads/2012/11/Resumen-del-impacto-de-la-reforma-sanitaria-en-elderecho-a-la-salud-de-la-poblaci\%C3\%B3n-inmigrante-Casos-documentados.sin-anexos-sin-casos.pdf [Accedido el 15 de julio de 2014]

MELERO, LUISA; DÍEZ, LUIS (2014): Claves contextuales para la detección de la violencia de género en mujeres inmigradas desde los servicios de Atención Primaria, Comunidad Valenciana: FundaciónCeiMigra. Disponible en:

http://www.ceimigra.net/observatorio/index.php/claves-contextuales-deteccionviolencia-genero[Fecha de acceso: 05 de julio de 2014]

MINISTERIO DE IGUALDAD (2010) Plan integral de lucha contra la trata de seres humanos con fines de explotación sexual. Disponible en: http://www.msssi.gob.es/ssi/violenciaGenero/tratadeMujeres/planIntegral/home htm [Fecha de acceso: 05 de julio de 2014]

MINISTERIO DE JUSTICIA (2013) Anteproyecto de Ley Orgánica para la Protección de la Vida del Concebido y de los Derechos de la Mujer Embarazada. Diciembre 2013. Disponible en: http://www.mjusticia.gob.es/cs/Satellite/es/1215198252237/ALegislativa P/12 88774452773/Detalle.html [Fecha de acceso: 05 de julio de 2014]

MINISTERIO DE SANIDAD, SERVICIOS SOCIALES E IGUALDAD (2012) Macroencuesta de violencia de género 2011. Disponible en: http://www.observatorioviolencia.org/upload images/File/DOC1329745747 m acroencuesta2011 principales resultados-1.pdf [Fecha de acceso: 05 de julio de 2014]

MINISTERIO DE SANIDAD, SERVICIOS SOCIALES E IGUALDAD (2013): Estrategia nacional para la erradicación de la violencia contra la mujer (2013 - 2016) Disponible en:

https://www.msssi.gob.es/ssi/violenciaGenero/EstrategiaNacional/home.htm [Fecha de acceso: 05 de julio de 2014]

MINISTERIO DE SANIDAD, SERVICIOS SOCIALES E IGUALDAD (2013): Interrupción Voluntaria del Embarazo. Datos definitivos correspondientes al año 2012. Madrid, Dirección General de Salud Pública, Calidad e Innovación. Disponible en:

https://www.msssi.gob.es/profesionales/saludPublica/prevPromocion/embarazo/docs/IV E 2012.pdf [Fecha de acceso: 05 de julio de 2014]

NACIONES UNIDAS (1981):Convención sobre la Eliminación de todas las formas de 
Discriminación contra la Mujer (CEDAW) Disponible en:

www.un.org/womenwatch/daw/cedaw/text/sconvention.htm [Fecha de acceso: 20 de diciembre de 2014]

NACIONES UNIDAS (2013):Informe del Relator Especial sobre las formas contemporáneas de racismo, discriminación racial, xenofobia y formas conexas de intolerancia, MutumaRuteere. Disponible en:http://daccess-ddsny.un.org/doc/UNDOC/GEN/G13/144/81/PDF/G1314481.pdf?OpenElement

[Fecha de acceso: 23 de mayo de 2014].

NOGUEIRA, JULIA (2014):Análisis de la legislación europea y española sobre salud sexual y reproductiva. Consecuencias de la reforma de la LO 2/2010 de salud sexual y reproductiva e interrupción voluntaria del embarazo. Fundación Alternativas. Disponible en:

http://www.falternativas.org/estudios-de-progreso/documentos/documentos-detrabajo/analisis-de-la-legislacion-espanola-y-europea-sobre-salud-sexual-yreproductiva-consecuencias-de-la-reforma-de-la-lo-2-2010 [Fecha de acceso: 23 de junio de 2014]

OBSERVATORI DE BIOĖTICA I DRET (2014) ¿Sigue siendo la salud un derecho fundamental en España? Revista de Bioética y Derecho, núm. 31, mayo 2014, p. 1-2.

OFICINA DEL ALTO COMISIONADO DE LAS NACIONES UNIDAS PARA LOS DERECHOS HUMANOS (2014): Experta de la ONU pide a España "un auténtico compromiso con la protección de los derechos humanos para todos". Disponible en:

http://www.ohchr.org/SP/NewsEvents/Pages/DisplayNews.aspx?NewsID=14545\&Lang $\underline{\mathrm{ID}=\mathrm{S}}$ [Fecha de acceso: 15 de junio de 2014].

Real Decreto-ley 16/2012, de 20 de abril, de medidas urgentes para garantizar la sostenibilidad del Sistema Nacional de Salud y mejorar la calidad y seguridad de sus prestaciones. Disponible en:

http://www.boe.es/boe/dias/2012/04/24/pdfs/BOE-A-2012-5403.pdf [Fecha de acceso: 15 de julio de 2015]

Real Decreto 1192/2012, de 3 de agosto, por el que se regula la condición de asegurado y de beneficiario a efectos de la asistencia sanitaria en España, con cargo a fondos públicos, a través del Sistema Nacional de Salud. Disponible en:

http://www.boe.es/diario boe/txt.php?id=BOE-A-2012-10477 [Fecha de acceso: 15 de julio de 2014]

Real Decreto 576/2013, de 26 de julio, por el que se establecen los requisitos básicos del convenio especial de prestación de asistencia sanitaria a personas que no tengan la condición de aseguradas ni de beneficiarias del Sistema Nacional de Salud y se modifica el Real Decreto 1192/2012, de 3 de agosto, por el que se regula la condición de asegurado y de beneficiario a efectos de la asistencia sanitaria en España, con cargo a fondos públicos, a través del Sistema Nacional de Salud. Disponible en:

http://www.boe.es/diario_boe/txt.php?id=BOE-A-2013-8190[Fecha de acceso: 15 de julio de 2014] 
SERVEI D'INFORMACIÓ I ESTUDIS (2011):Anàlisi de l'impacte de la immigració en les interrupcions voluntàries de l'embaràs a Catalunya. Barcelona. Departament de Salut. Generalitat de Catalunya. Disponible en:

http://www20.gencat.cat/docs/salut/Home/El\%20Departament/Estadistiques\%20sanitari es/Dades\%20de\%20salut $\% 20$ i $\% 20$ serveis $\% 20$ sanitaris/Interrupcio\%20voluntar ia\%20de\%20lembaras\%20(IVE)/documents/immigracio ives.pdf[Fecha de acceso: 22 de junio de 2014]

VVAA (2012):Estrategias para Combatir la Doble Violencia Contra las Mujeres Migrantes Irregulares Proteger sus Derechos y Asegurar la Justicia. Bruselas: PICUM Disponible en:

http://www.migrarconderechos.es/bibliografia/combatir_doble_violencia_contra_mujer es migrantes [Fecha de acceso: 15 de julio de 2014]

YO Sí SANIDAD UNIVERSAL (2013):Un año de exclusión sanitaria, un año de desobediencia. Campaña de desobediencia al Real Decreto-Ley 16/2012. Disponible en:http://lapasucat.blogspot.com.es/p/documentacio.html[Fecha de acceso: 20 de junio de 2014]

YO SÍ SANIDAD UNIVERSAL, SOCIEDAD MADRILEÑA DE MEDICINA DE FAMILIA Y COMUNITARIA(2014): Informe REDES Registro Estatal para la Denuncia de la Exclusión Sanitaria. Disponible en:

http://yosisanidaduniversal.net/media/blogs/materiales/Informe\%20REDES.pdf?mtime =1412027824[Fecha de acceso: 20 de diciembre de 2014] 